\title{
THERMAL BIFURCATION OF THE OUTER PHOTOSPHERE
}

\author{
T. R. AYRES \\ Center for Astrophysics and Space Astronomy \\ University of Colorado \\ Boulder, Colorado, USA
}

\begin{abstract}
The degree of thermal heterogeneity at the base of the solar chromosphere is substantially beyond that simulated in the best-available multicomponent models; casting serious doubts on inferences drawn from them.
\end{abstract}

Everyone knows that the chromosphere of the Sun is hot. After all, spatially-averaged profiles of strong optical and ultraviolet resonance lines - $\mathrm{Ca} \mathrm{II} \mathrm{H} \mathrm{and} \mathrm{K}, \mathrm{Mg} \mathrm{II} h$ and $k$, and H I Ly $\alpha$, for example - clearly show prominent emission reversals in their cores, indicating a temperature inversion at the top of the photosphere (e.g., Athay 1976). Virtually all empirical models of the solar outer atmosphere have incorporated such a temperature inversion. The most complete such model - that of Vernazza, Avrett, and Loeser (1981) - presents a range of thermal profiles to simulate spatial inhomogeneities. Nevertheless, each of the six distinct components has a temperature inversion at about the same altitude $\left(\approx 500 \mathrm{~km}\right.$ above $\left.\tau_{5000}=1\right)$. The multi-component model successfully reproduces a wide range of spectral diagnostics - lines and continua - broadly covering the electromagnetic spectrum. The principal use of the model has been to calculate the radiative cooling as a function of altitude in the chromosphere, against which to compare the predictions of various mechanical heating scenarios.

Everyone knows that the chromosphere is hot, but somebody apparently neglected to tell the infrared bands of carbon monoxide! Initially, measurements of the strong $\Delta v=1$ transitions (near $2150 \mathrm{~cm}^{-1}=4.7 \mu \mathrm{m}$ ) by Noyes and Hall (1972) revealed low core brightness temperatures $\left(T<4200 \mathrm{~K}\right.$; compared with $T_{\min } \gtrsim 4400$ deduced from $\mathrm{Ca}$ II and $\mathrm{Mg}$ II) in the most opaque of the vibration-rotation lines; later, Ayres and Testerman (1981) confirmed the earlier results using the newly-commissioned Fourier transform spectrometer on the McMath telescope at Kitt Peak. At the extreme limb, where radition emerges from the highest accessible levels of the atmosphere in a given diagnostic, the strongest $\mathrm{CO}$ absorptions indicated the existence of very cool plasma $(T<3800 \mathrm{~K})$ and no hint of any chromospheric $(T>6000 \mathrm{~K})$ material, contrary to the expectations of the best-available homogeneously stratified models. Figure 1 illustrates the type of thermal profile derived from high temporal resolution and moderate spatial resolution recordings of the infrared $\mathrm{CO}$ bands in activity-free areas at disk center and near the extreme limb. The vertical 


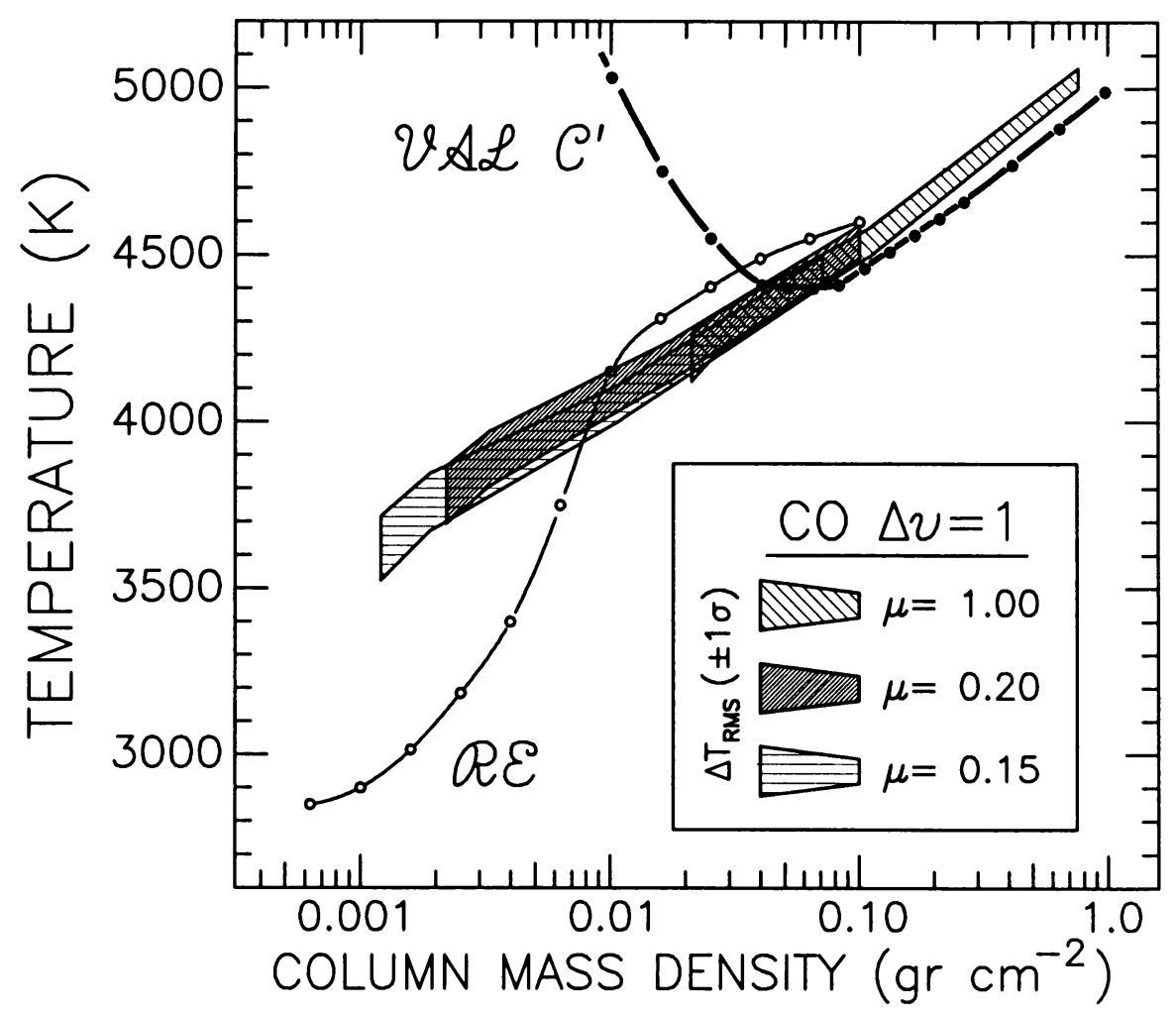

Figure 1: Measured CO brightness temperatures compared with an empirical chromospheric model (the VAL $C^{\prime}$ model of Maltby et al. [1986]), and a theoretical temperature profile calculated in radiative equilibrium including $\mathrm{CO}$ cooling (Anderson and Athay 1989).

extents of the shaded areas depict the maximum rms thermal fluctuations inferred to exist at the different levels of the atmosphere over horizontal size-scales comparable to a $p$-mode wavepacket (several $\mathrm{Mm}$ ). The large response of the $\mathrm{CO}$ bands to $p$-mode excitations indicate a high altitude of formation.

The inescapable conclusion was that the "chromosphere" of the Sun must contain substantial amounts of relatively cool material in addition to the classical hot gas that one ordinarily associates with it. In the Ayres and Testerman model (later quantified by Ayres, Testerman, and Brault 1986) the weak central reversals in the cores of the $\mathrm{Ca} I I$ lines, for example, might arise as a mixture of strong emission from small, intensely-heated regions diluted by pure absorption profiles from the more extensive cool component.

The juxtaposition of hot and cold gas in the chromosphere was attributed by Ayres (1981) to a thermal instability driven on the low-temperature side by the powerful surface 
cooling of the near-LTE CO $\Delta v=1$ bands, and on the high-temperature side by strong radiative emission in the UV resonance lines of abundant species (e.g., $\mathrm{Mg} \mathrm{II}, \mathrm{Ca} \mathrm{II,} \mathrm{and} \mathrm{H}$ I). The low-temperature cooling is enhanced for decreasing temperatures below about 4500 $\mathrm{K}$ by the exponentially increasing formation of the $\mathrm{CO}$ molecules, and stabilizes when the molecules have exhausted the available atomic carbon $(T \lesssim 4000 \mathrm{~K})$. The high-temperature cooling is promoted as $T$ rises above about $5000 \mathrm{~K}$ by the exponential thermal sensitivity of the electron collisional excitation rates, as well as by the increasing population of electrons due to hydrogen ionization. However, in the intermediate range $4000 \lesssim T \lesssim 5000$ neither the CO infrared bands nor the atomic UV resonance lines are effective coolants. Thus, the plasma cooling function at chromospheric heights exhibits two distinct stable phases, with an unstable intermediate temperature range.

The bifurcation of the cooling function leads to a thermal instability when the chromospheric gas is subjected to a critical level of nonradiative heating: the gas remains cool and near the radiative equilibrium stratification - when the mechanical heating falls below the critical value; but reverts to the hot, classical chromospheric phase when the nonradiative heating exceeds it. Ayres, Testerman, and Brault (1986) modelled simultaneous observations of the $\mathrm{CO}$ bands and $\mathrm{Ca}$ II $\mathrm{K}$ in quiet regions on the solar disk and in areas strongly disturbed by magnetic activity. The authors concluded that only a small fraction ( $\lesssim 10 \%)$ of the solar chromosphere in quite regions is strongly-enough heated to produce hot plasma; and even in magnetic active regions only perhaps $\approx 50 \%$ of the surface is truly chromospheric. Thermal inhomogeneities of that magnitude, particularly in the quiet Sun, are enough to invalidate most of the previous homogeneously-stratified models based on spatially-average solar spectra (or integrated starlight), and cause us to reexamine our understanding of chromospheric structure not only on the Sun, but also on the other stars of late spectral type.

Figure 2 illustrates more graphically the structural organization of the solar atmosphere. At the altitude of the " $T_{\min }$ ", the chromosphere consists of two fundamental types of structures, both of small surface coverage: (1) long-lived network bright points, whose heating very likely is magnetic in origin; and (2) transient cell-interior flashes, whose heating might be electrodynamic as well, although the disturbances could possibly be purely acoustic. An excellent discussion of the two distinct types of bright points has been provided by Cram (1985). In the quiet Sun, the BPs cover perhaps $<20 \%$ of the surface (dominated by the cell-interior BPs in area, although the network points are brighter in $\mathrm{Ca}$ II); but in active regions the coverage might reach as much as $60 \%$ (dominated by the magnetic BPs). The (large) remaining volume at, and immediately above, the $T_{\min }$ (up to perhaps $700 \mathrm{~km}$ ) is occupied by material that is substantially cooler than that in the heated structures. One might think of the cool gas as organized into $\mathrm{CO}$ clouds. The clouds continually form, perhaps through the adiabatic cooling of gas advected to high altitudes by convective overshoot, or by supergranular plumes (e.g., November 1989); and continually are disrupted by the sporatic disturbances responsible for the cell flashes.

Recent Non-LTE blanked models of the solar chromosphere with prescribed heating have pointed to the $T_{\min }$ region as the critical location of maximum nonradiative energy 


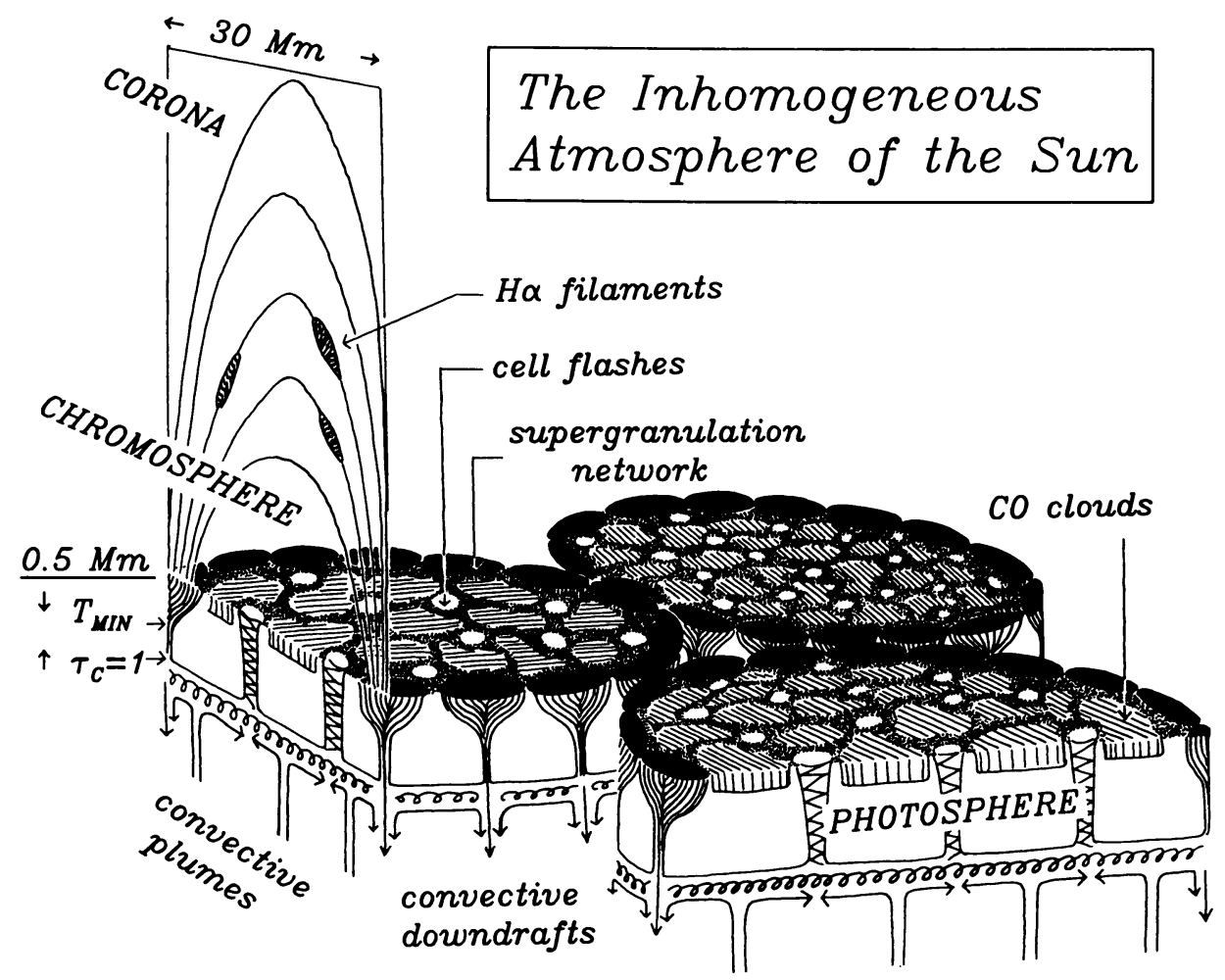

Figure 2: The inhomogeneous atmosphere of the Sun.

deposition (Anderson and Athay 1989). It is there that we have the best chance to isolate the elusive chromospheric heating mechanism. But, if the $T_{\min }$ in fact is dominated by cool structures, then the spatially-averaged thermal profiles derived by Anderson and Athay, and earlier by VAL, have little meaning. Instead, the energy-balance modelling should be focused on the specific structures - network and cell-interior BPs - where the strongly-heated gas at the base of the chromosphere truly resides. Of course, the issue is further complicated by the fact that the rapidly diverging fields of the network magnetic filaments must fill all of the available volume in the chromosphere above some critical level (the CO "clouds" have a small vertical scale height owing to their depressed temperatures). Thus, the middle chromosphere (at an altitude of about $1000 \mathrm{~km}$ ) might be more thermally homogeneous than the underlying $T_{\min }$ region. Nevertheless, $\mathrm{Ca} \mathrm{II} \mathrm{K}_{2}$ and Ly $\alpha$ filtergrams - characteristic of those high levels - exhibit considerable structure, and the network is clearly recognizable even at C IV temperatures $\left(10^{5} \mathrm{~K}\right)$.

Critically needed is a major effort to define a grid of thermally-distinct temperature profiles to more accurately describe the true physical conditions at the critical interface between photosphere and chromosphere. Such a grid of models can be used to explore 
the nature of the nonradiative heating mechanism in a more favorable light than present thermal profiles, which ignore the important role of cool gas in the low chromosphere.

ACKNOWLEDGEMENTS. This work was supported by grants from the National Science Foundation and the National Aeronautics and Space Administration. The observations described in Fig. 1 were obtained at the Kitt Peak facility of the National Solar Observatories, operated by AURA under contract to the NSF.

\section{REFERENCES}

Anderson, L. S. 1989, Ap. J., 339, 558.

Anderson, L. S., and Athay, R. G. 1989, "Model Solar Chromosphere with Prescribed Heating", Ap. J., (submitted).

Athay, R. G. 1976, The Solar Chromosphere and Corona: Quiet Sun, (Dordrecht: D. Reidel).

Ayres, T. R. 1981, Ap. J., 244, 1064.

Ayres, T. R., and Testerman, L. 1981, Ap. J., 245, 1124.

Ayres, T. R., Testerman, L., and Brault, J. W. 1986, Ap. J., 304, 542.

Cram, L. 1987, in Cool Stars, Stellar Systems, and the Sun, (eds.) J. L. Linsky and R. E. Stencel (New York: Springer-Verlag), p. 123.

Maltby, P., Avrett, E. H., Carlsson, M., Kjeldseth-Moe, O., Kurucz, R. L., and Loeser, R. 1986, Ap. J., 306, 284.

November, L. J. 1989, "The Vertical Component of the Supergranulation Convection", Ap. J., (submitted) [NOAO preprint No. 257].

Noyes, R. W., and Hall, D. N. B. 1972, Ap. J. (Letters), 176, L89.

Vernazza, J. E., Avrett, E. H., and Loeser, R. 1981, Ap. J. Suppl., 45, 635. 